\title{
Actual trends in the use of the kastle-meyer test: applications in different species and verification of the limit of detection of sensitivity and vestigiality
}

\begin{abstract}
Forensic Science is formed by areas that perform analysis in different traces found in crime scenes and that can correlate with the fact that happened, thus being able to help in judicial procedure. One of the most important evidence found at a crime scene is blood, but to prove it, rapid tests are performed, such as the Kastle-Meyer (KM) test, which is widely used nowadays because it is considered a fast, cheap and efficient test. Its principle is based on the decomposition of hydrogen peroxide by the hemoglobin present in the blood in water and oxygen, changing the color of the substrate, along with the presence of phenolphthalein, from colorless to pink. This test is well characterized in human blood, but there are no reports referring to its efficiency in blood from animals of other species, which is of extreme relevance, since there are differences in relation to hemoglobin of different species and, still, the number of reports against animal abuse have grown in last years. Thus, the objective of this study was to evaluate the efficiency, sensitivity and vestigiality of the Kastle-Meyer test in human, dog and cat blood.
\end{abstract}

The results showed that, since the three species studied were mammals, the test was efficient in detecting the presence of blood, as well as, in relation to sensitivity, for human and canine blood the test was able to detect to be blood until the dilution of 1:10.000, while for cats the reagent showed positive results up to the dilution of 1:1.000. In relation to the vestigiality, different products such as water, sanitary water, $70^{\circ}$ alcohol, anionic surfactant and quaternary ammonia were used to clean the blood and then the reagent was tested, which presented positive results for the three species used. Thus, it is possible to conclude that the KM reagent is an important spot test that can be used in crime scenes both to detect the presence of human blood, and for domestic animals such as dogs and cats. In addition, in the case of an unpreserved area, the test was also shown to be effective in providing positive results for different blood dilutions and for latent blood.

Keywords: blood, efficiency, forensic, kastle-meyer test, sensitivity,trait
Volume 8 Issue 4 - 2019

\author{
Renata Inzinna Bernardo Fonseca, ${ }^{2}$ Esther \\ Lopes Ricci, ${ }^{2}$ Helenice de Souza Spinosa,' \\ Maria Marta Bernardi, ${ }^{5}$ Gabriel Ramos \\ de Abreu,' Paula A Faria Waziry, ${ }^{3}$ Maria \\ Aparecida Nicoletti, ${ }^{4}$ Simone Rodrigues \\ Ambrosio, ${ }^{6}$ lago Portolani de Araujo, ${ }^{6}$ \\ Juliana Weckx Peña Muñoz, ${ }^{6}$ André Rinaldi \\ Fukushima' \\ Department of Pathology, School of Veterinary Medicine and \\ Animal Science, University of São Paulo, São Paulo, Brazil \\ ${ }^{2}$ Health Science Institute, Presbiterian Mackenzie University, São \\ Paulo, Brazil \\ ${ }^{3}$ Kiran C Patel College of Osteopathic Medicine, Nova \\ Southeastern University, Fort Lauderdale, Florida, United States \\ of America \\ ${ }^{4}$ Faculty of Pharmaceutical Sciences, University of São Paulo, São \\ Paulo, Brazil \\ ${ }^{5}$ Institute of Ciences of Health University Paulista, Indianópolis \\ São Paulo/SP, Brasil \\ ${ }^{6}$ São Judas Tadeu University, São Paulo, Brazil
}

Correspondence: André Rinaldi Fukushima, Department of Pathology, School of Veterinary Medicine and Animal Science, University of São Paulo, São Paulo, Orlando Marques de Paiva, 87, 05508-270, Brazil, Email fukushima@usp.br

Received: July 13, 2019 | Published: July 22, 2019

\section{Abbreviations}

KM, Kastle-Meyer

\section{Introduction}

Forensic, Latin means forum, which in Roman times referred to a market where commercial transactions and legal proceedings were conducted and, after several years in which the term was used only for legal investigations, has often become used for any detailed analysis in a search for evidence. ${ }^{1}$ The spot tests are a form of analysis carried out by field experts at crime sites that are fundamental for identification and confirmation of traces for later analysis by the laboratory expert, and the relation with crime is confirmed, this is called evidence. ${ }^{2}$ Since blood is the most commonly found physical vestige at a crime scene, several tests have been developed to determine if a reddish patch is actually blood or not. The rapid test of benzidine has been used for a long time, in which the positive result for the presence of blood is presented through the appearance of the blue coloration, however, several false positive results have been detected and, moreover, due to the carcinogenic characteristics in its composition, began to be questioned and later replaced. ${ }^{3-5}$ An important rapid test that has been widely used to identify the presence of blood is the Kastle
Meyer reactive, which has the characteristic of being fast, efficient and cheap, facilitating its access and use. The Kastle-Meyer test was discovered between 1901 and 1903 and after years of improvement it was used for medical-legal purposes. Its principle is based on the decomposition of hydrogen peroxide (revealing reagent), which reacts with the peroxidase residues of hemoglobin and reacts with phenolphthalein, making the medium pink if there is blood, as can be observed in (Figure 1). ${ }^{6-8}$

Cox (1991) has shown in his study evaluating the presumptive Kastle-Meyer, benzidine, malachite green and ortho-tholidine tests that the phenolphthalein test is best because of its sensitivity of 1 : 10.000 for blood and also for the blood its negative result for plant peroxidase, which did not occur in the other tests. Furthermore, according to Cox (1991) and Higaki and Philip (1976), the presumptive Kastle-Meyer test is very effective in detecting latent blood, mainly due to its sensitivity. In addition to the existence of crimes against the person, there is an increasing number of complaints around the world due to mistreatment of animals. In Brazil, there is specific legislation that portrays the importance of crimes against fauna, as these may cause environmental imbalance, in addition to violating Law No. 9605 of 1998, which in article 32 defines mistreatment as the insult that, even without lesions in the animal, can cause suffering. ${ }^{9-11}$ Thus, 
it is important to highlight the scarcity of work related to the analysis of crime sites where animal maltreatment may have occurred and that it is therefore essential to understand whether tests such as KastleMeyer, which is a rapid test considered efficient and inexpensive, is able to detect the presence of blood of non-human species as well as latent or even diluted blood, since often crime scenes are not found preserved. ${ }^{12}$

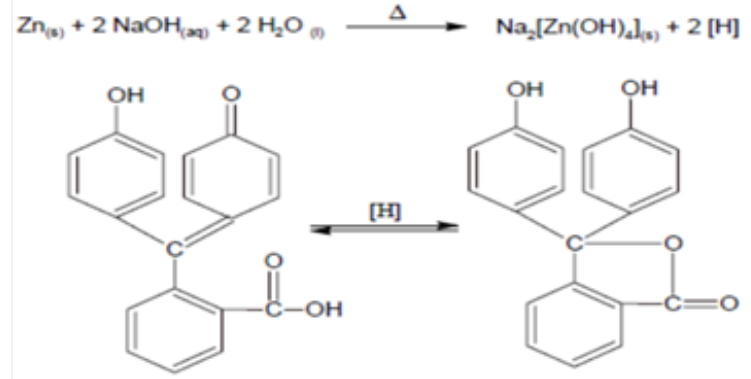

red conformation

colorless conformation
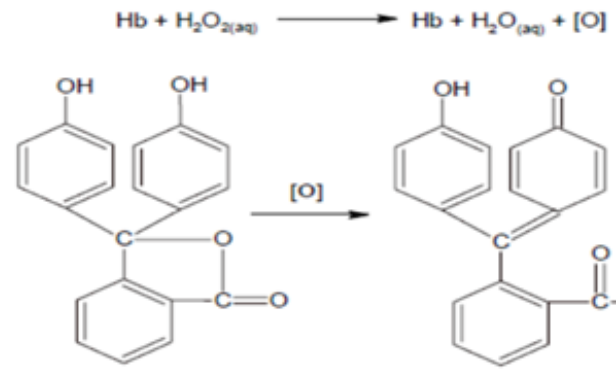

colorless conformation

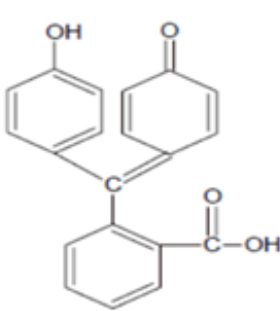

red conformation

\section{Sensitivity test}

To evaluate the sensitivity of the KM reagent, a 96 well plate was used in which the blood was diluted in distilled water in the following concentrations: $1: 10,1: 100,1: 1000,1: 10000,1: 100.000$ and 1 : 1000000 and then subjected to the test procedure.

\section{Vestigiality test}

Also, since it is recurrent to find crime scenes that tried to be cleaned, the Kastle-Meyer test was also validated on surface after contamination with blood and later cleaning with common water, bleach, $70^{\circ}$ alcohol, anionic and quaternary tensioactive ammonia.

\section{Results}

After the preparation of the Kastle-Meyer reagent and the three day wait the tests were started. First, the specificity test was performed to verify that the reagent would be able to identify blood from the different species. To demonstrate this result, photos of the swab were taken after addition of blood, water and the reagent as shown in Figures $2,3 \& 4$ below.

A

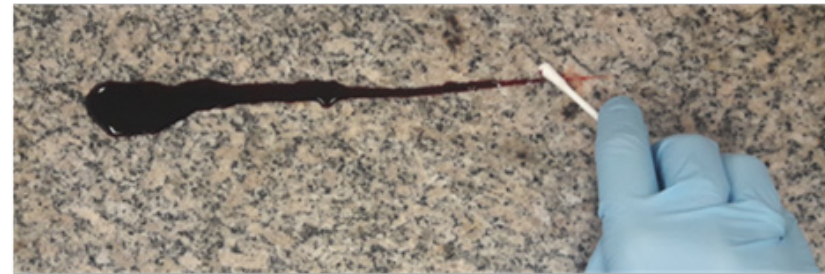

B

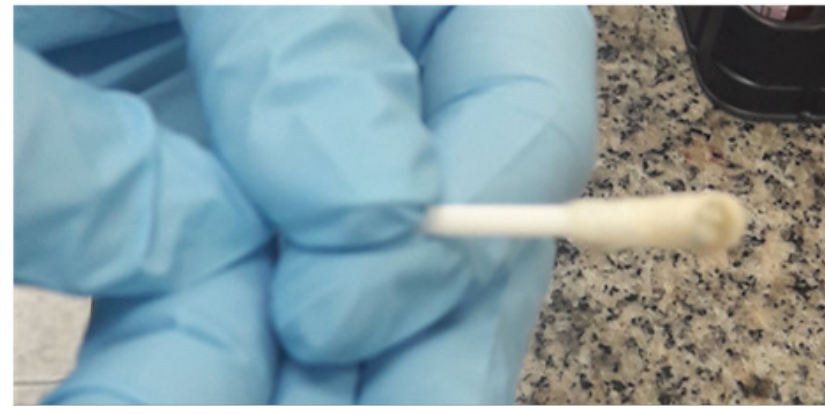

Figure 2 (A) Swab being passed on human blood; (B) positive result for blood.

A

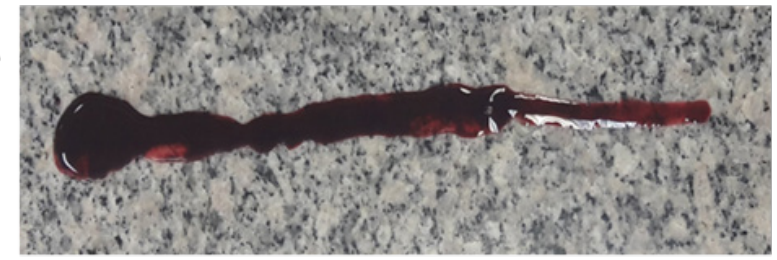

A solution of sodium hydroxide $(2 \mathrm{~g}$ of $\mathrm{NaOH}$ added to $9 \mathrm{ml}$ of distilled water) was added and $100 \mathrm{mg}$ of phenolphthalein dissolved in $1 \mathrm{ml}$ of ethanol was added, then $2 \mathrm{~g}$ of metal zinc powder was added to the solution and subjected to a sheet of heating at $80^{\circ} \mathrm{C}$ under constant stirring until the coloration disappears. As a precaution, 3 days of rest were recommended so that the intensity of the color disappeared completely. ${ }^{13}$

\section{Efficiency test}

To analyze the ability of the KM reagent to detect the presence of human, canine and feline blood, the blood samples were individually arranged on a smooth non-porous surface and then a flexible rod was moistened with saline and the sample followed by one drop of the Kastle-Meyer reagent and finally one drop of hydrogen peroxide $(5 \%)$.

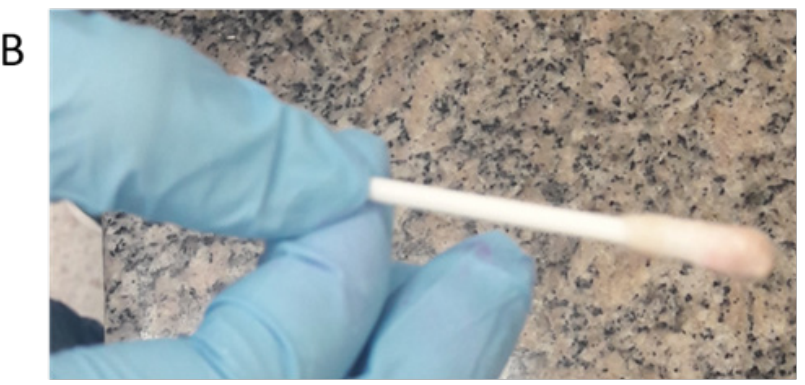

Figure 3 (A) Dog blood on the bench; (B) positive result on swab. 
A

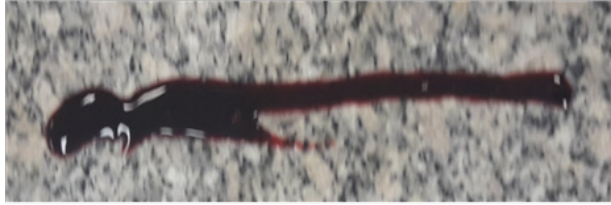

B

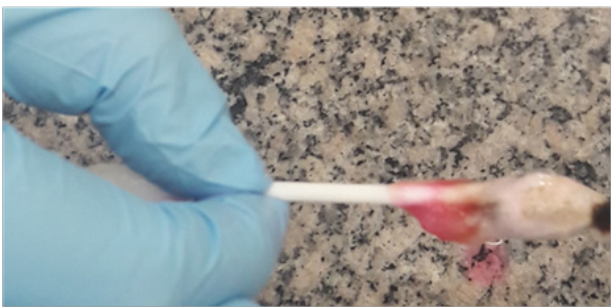

As can be observed, the three species tested showed a positive result after contact with whole blood, Kastle-Meyer reagent and distilled water. Then, a 96-well plate was used to perform the sensitivity test on each species. To this end, whole blood was added to the different lines of the first column of the plate and in the following distilled water was added to serially dilute the blood of the different species in 1:10, 1: 100, 1: 1,000, 1: 10,000,1:100,000 and 1: 1,000,000. The result is shown in Figures 5, 6 \& 7 below.

By analyzing the results of the sensitivity test, it can be seen that the Kastle-Meyer reagent is capable of detecting human and dog blood up to the dilution of 1: 10,000, while for the cat it was only detectable to be blood up to the dilution of 1: 1,000. Furthermore, in all three species, the reagent did not detect the presence of blood in the 1: 1,000,000 dilution. Afterwards, the plasma and serum of dog and cat were used to test the sensitivity (Figure 8).

Figure 4 A) Cat's blood on the bed; (B) positive result for blood in the swab.

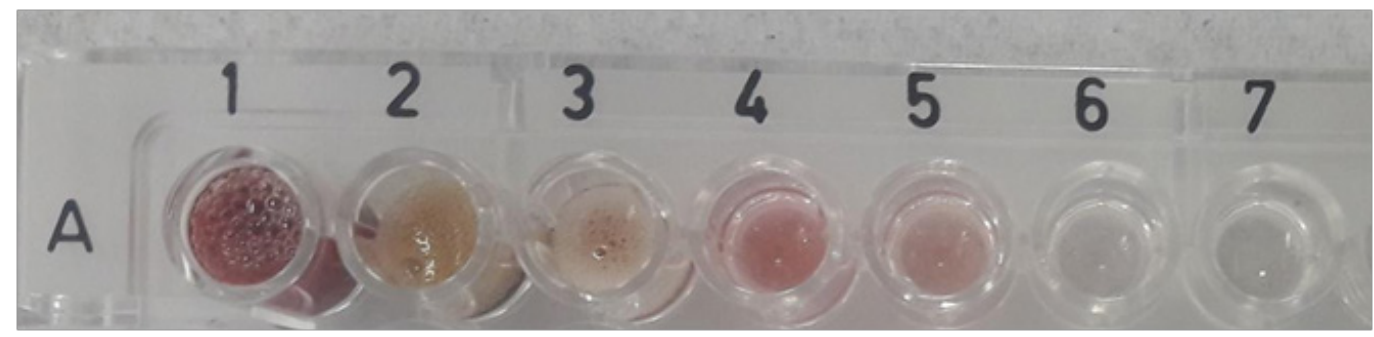

Figure 5 Human blood.

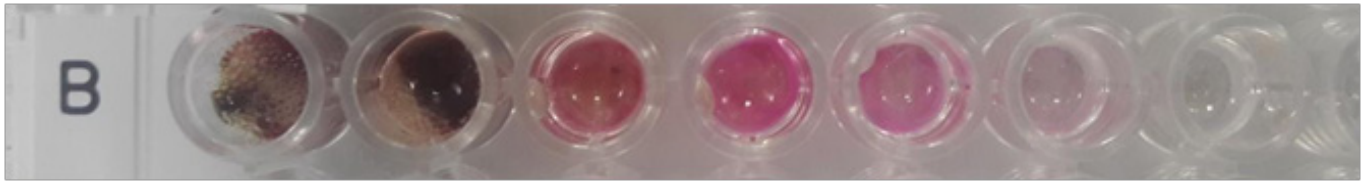

Figure 6 Dog's blood.

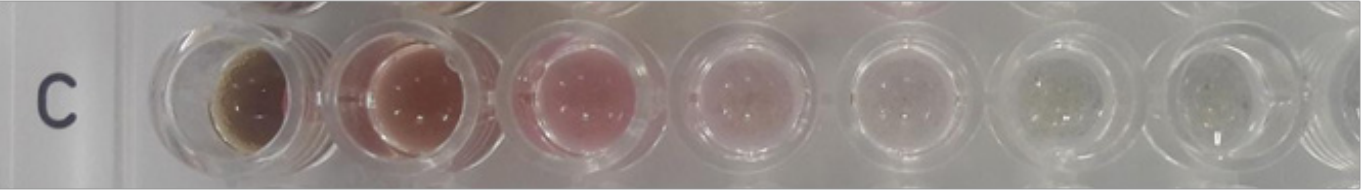

Figure 7 Cat's blood.

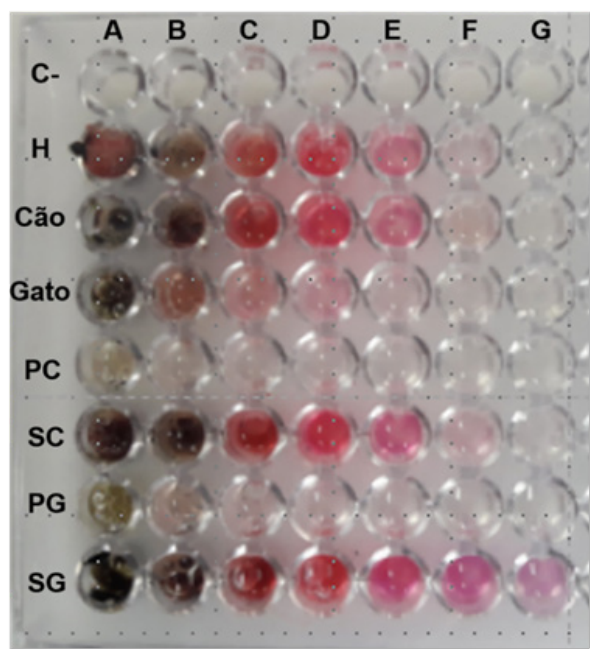

Figure 896 wells plate with sensitivity test for blood, serum and plasma. C, negative control; H, human; PC, plasma dog; SC, Serum; PG, plasma cat; SG, serum cat.
Through this test it was possible to observe that both when analyzing the reagent in the plasma of dog or cat it is not possible to observe a negative result. When the serum is observed, the Kastle-Meyer reagent is able to detect the presence of blood up to the dilution of 1: 10,000 for the dog and up to 1: 1,000,000 for feline blood. Also, it is important to note that a negative control was used in the first row of the water-only plate to verify the ability of the Kastle-Meyer test to distinguish the positive from the negative. Afterwards, blood was used to perform the test called vestigiality, that is, the ability of the reagent to identify blood from a smooth and non-absorbent surface after cleaning with different materials such as
i. Water
ii. Hypochlorite
iii. Alcohol $70^{\circ}$
iv. Anionic surfactant and
v. Quaternary ammonia

In (Figure 9) the result of human blood is observed, while in (Figures $10 \& 11$ ) the dog and cat blood respectively. 

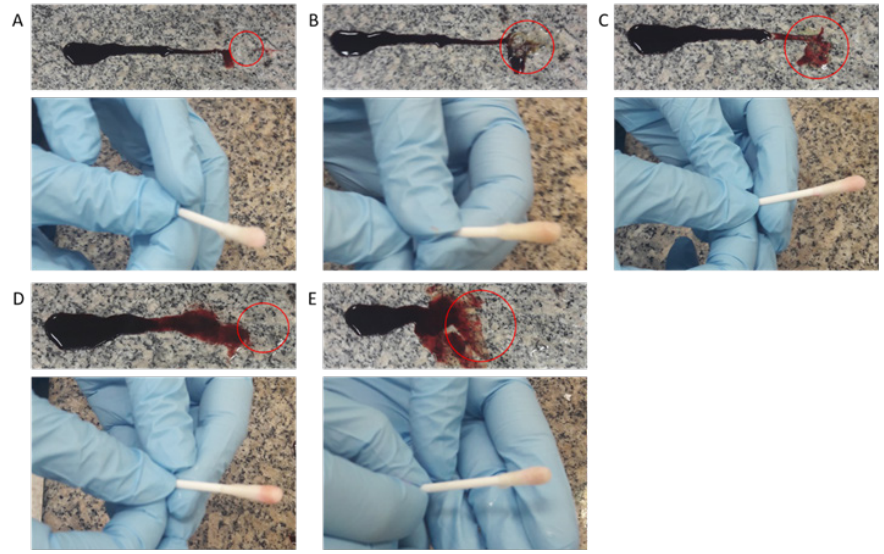

Figure 9 Human blood - (A) blood cleansing with water and swab. (B) cleaning the blood with hypochlorite and swab. (C) blood cleaning with $70^{\circ}$ alcohol and swab. (D) blood cleansing with anionic surfactant and swab. (E) blood cleansing with quaternary ammonia and swab.
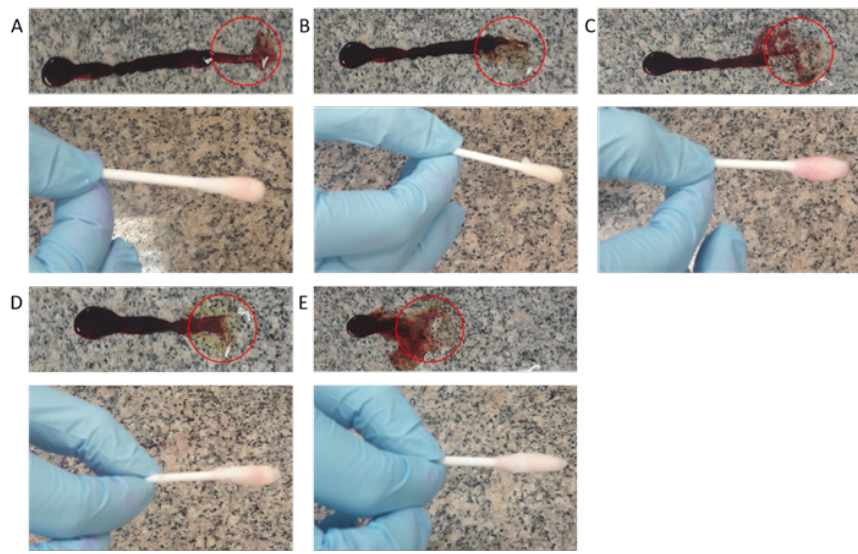

Figure 10 Dog blood - (A) blood cleansing with water and swab. (B) cleaning the blood with hypochlorite and swab. (C) blood cleaning with $70^{\circ}$ alcohol and swab. (D) blood cleansing with anionic surfactant and swab. (E) blood cleansing with quaternary ammonia and swab.
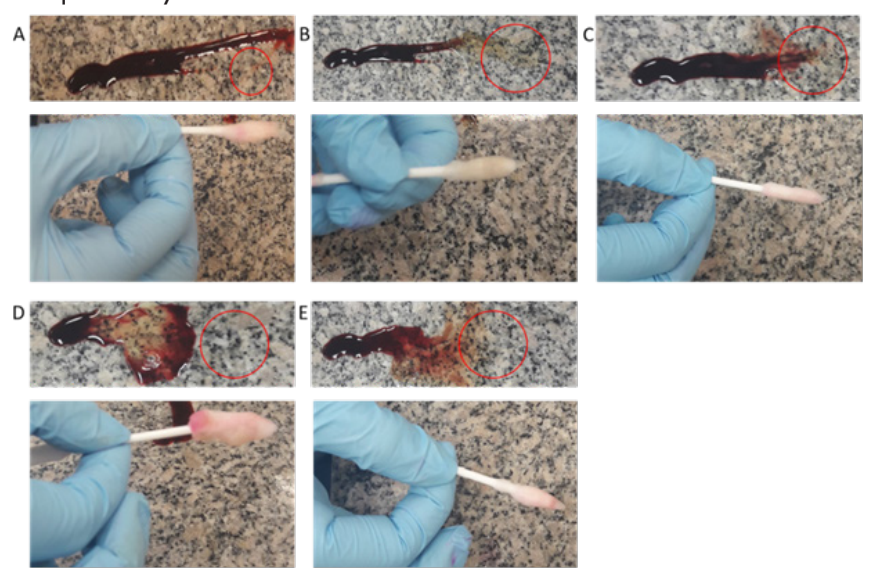

Figure I I Cat Blood - (A) Blood cleansing with water and swab. (B) cleaning the blood with hypochlorite and swab. (C) blood cleaning with $70^{\circ}$ alcohol and swab. (D) blood cleansing with anionic surfactant and swab. (E) blood cleansing with quaternary ammonia and swab.

The vestigiality test showed that when blood of a human is cleansed with the above-mentioned different products shown in the images by the red circle, the Kastle-Meyer reagent is still able to identify the presence of blood by changing the color of the swab from colorless to pink. When canine blood is used for the experiment, a change in the color of the swab after surface cleaning and cleaning with the different products is evident, evidencing the effectiveness of the Kastle-Meyer test to identify the presence of occult blood. In the case of feline blood, it was observed after cleaning the surface and subsequent application of the test, that the result was positive for blood, and more clearly than when compared to the human and dog bloods previously demonstrated.

\section{Discussion}

For this work, a test was first carried out to guarantee its specificity. The experiment was carried out on human, dog and cat blood samples, in which the reagent was efficient in detecting the three samples. This result was expected, since it is mammalian species, that is, the blood contains anucleated red blood cells, with residue of the enzyme peroxidase, that are able to decompose the hydrogen peroxide, generating the pink color, consistent with the mechanism chemical reactions of the Kastle-Meyer reactive. ${ }^{8,14}$ In addition, an analysis was performed to validate and measure the sensitivity of the test for the three samples in question. The negative control did not show changes in its coloration either at the time of addition of the hydrogen peroxide or after 10 minutes, differently from what was observed by Tobe et al., Who after four minutes of the addition of $\mathrm{H}_{2} \mathrm{O}_{2}$ obtained pink coloration. ${ }^{15}$ The chemical mechanism of the Kastle-Meyer reaction requires enzymatic activation intermediated by the decomposition of hydrogen peroxide into reactive oxygen species in order to generate the breakdown of the aromatic ring, thus forming in the chemical structure of phenolphthalein a carboxylic acid, which by in turn, promotes resonance in its chemical structure and consequently color change, so our findings show that our negative control was not contaminated with reactive oxygen species, unlike the negative control of Tobe et al. ${ }^{8}$

Also according to the results presented here through the sensitivity test, the Kastle-Meyer Reagent was able to detect the presence of blood in the samples up to the dilution of 1:10,000 in human and dog, but not in 1: 100,000, as in previously described results, while cat blood was detectable up to a 1: 1,000 dilution. When tested on surfaces such as filter paper or cotton clothing, the Kastle-Meyer test showed a positive result in dilutions up to $1: 10,000$, corroborating with the present study. ${ }^{3,15,16} \mathrm{Cox}^{16}$ demonstrated in his study evaluating the presumptive Kastle-Meyer, benzidine, malachite green and orthotholidine tests and observed that phenolphthalein is the best because of its sensitivity of 1: 10,000 for blood and also for the blood its negative result for plant peroxidase, which did not occur in the other tests. The Kastle-Meyer reagent was also applied to blood plasma of dogs and cats, that is, blood without the presence of red blood cells, white blood cells and platelets, containing only water, nutrients, salts, coagulating factors, among and, as expected, the result was negative for the presence of blood, since without the red blood cells, there is no hemoglobin and consequently no chemical reaction is necessary to identify the presence of blood. ${ }^{5}$ However, when the test was used in the blood containing serum, that is, in the presence of an anticoagulant, preventing the wall of the erythrocyte from rupturing when it came into contact with the external environment, the result was positive for the presence of dog blood until the dilution of 1: 10.000 and, for cat, to the last dilution, i.e., 1: 1.000 .000 . In the vestigiality test, it was determined that this would be performed on a non-absorbent and smooth surface, since false-positive results for the Kastle-Meyer test on jeans have already been described, for example, due to the use of 
peroxidases in their manufacture. ${ }^{17}$ According to $\operatorname{Cox}^{16}$ and Higaki et al., ${ }^{3}$ the presumptive Kastle-Meyer test is very effective in detecting latent blood, mainly due to its sensitivity.

This result was corroborated in this experiment, since the reagent was able to detect the presence of blood after cleaning the surface with different products, such as water, hypochlorite, $70 \%$ alcohol, a common detergent and the quaternary ammonia and, not only of human blood, but also animal blood of cat and dog. The cellular, protein-enzymatic framework in the interspecies hematological area configures differences that can vary from insignificant to extremely relevant. The Kastle-Meyer test is based on the ability of catalase to convert peroxide into reactive oxygen species, thus promoting the conversion of phenolphthalein colorless for pink staining, therefore, these hematological-enzymatic differences may be strongly related to the difference in sensitivity in the tests performed with canine blood and feline blood, however, it is necessary to carry out tests to verify the enzymatic- hematological interspecies.

\section{Conclusions}

From the experiments performed it was possible to corroborate previous studies referring to the Kastle-Meyer test as a good presumptive test for the detection of blood from crime sites against the person or against the fauna, since it was possible to obtain a positive result for both blood human and animal blood, and also, at different dilutions, the reactive showed to be effective. In addition, it was also possible to validate the Kastle-Meyer reagent for its vestigiality, that is, its ability to detect latent blood, obtaining positive results for the presence of blood of the three species used. Although the KM test has shown a high presumptive power for detecting local and vestigial blood, it has limitations in use, and more studies are needed in the area of forensic hematology to understand in more depth the real reasons for which this test presented different interspecies results.

\section{Acknowledgments}

None.

\section{Conflicts of interest}

The author declares that there are no conflicts of interest.

\section{References}

1. Gunn A. Essential Forensic Biology. Second. Liverpool: WileyBlackwell, 2009.
2. Brennan N, Marcus D, Reno J. Crime Scene Investigation: A Guide for Law Enforcement. Washington. Epub ahead of print. 2013.

3. Higaki RS, Philp WMS. A study of the sensitivity, stability and specificity of phenolphthalein as an indicator test for blood. J Can Soc Forensic Sci. 1976;9(3):97-102.

4. An JH, Shin KJ, Yang WI. Body fluid identification in forensics. $B M B$ Rep. 2012;45:545-553.

5. James SH, Edel CF. Bloodstain Pattern Interpretation. Forensic Sciences: Introduction to Scientific \& Investigation Techniques. CRC Press. $1997 ; 176 \mathrm{p}$.

6. Glaister J. Kastle-Meyer test for detection of blood-The Kastle-Meyer test for the detection of blood considered from the medico-legal aspect. Br Med J. 1926;1(3406):650-652.

7. Gaensslen RE. Identification of blood. In: Frank r, editor. Forensic Serology, Immunology and Biochemistry. University of Michigan Library. 1983;71-145 p.

8. Chemello E. Forensic Science: Bloodstains. Virtual Chemistry. 2007;111.

9. Fukushima AR, Spinosa $\mathrm{H}$ de $\mathrm{S}$. Manual of toxicological forensic analysis focused on crimes against animals. São Paulo: Intertox. 2017;12(2).

10. Almeida JR. Judicial and insurance environmental expertise: impact, damage and environmental liability. Rio de Janeiro: Thex. 2006;501 p.

11. Levai LF. Animal Rights, 2nd ed. Campos do Jordão: Mantiqueira. 2004;159 p.

12. Eckert WG. Scientific Evidence in Court. Introduction to Forensic Sciences, 2nd ed. Florida: CRC Press. 1997;78 p.

13. Helmenstine AM. How to Make Kastle-Meyer Solution. ThoughtCo. 2017.

14. Hickman CP, Larson A, Roberts LS, et al. Internal fluids and breathing. In: Integrated Principles of Zoology. Rio de Janeiro: Guanabara Koogan S.A. $2004 ; 793$ p.

15. Tobe SS, Watson N, Daéid NN. Evaluation of six presumptive tests for blood, their specificity, sensitivity, and effect on high molecular-weight DNA. J Forensic Sci. 2007;52(1):102-109.

16. Cox M. A Study of the Sensitivity and Specificity of Four Presumptive Tests for Blood. J Forensic Sci. 1991;36(6):1503-1511.

17. Lalonde W, Millman JS. Case study: Loss of Kastle-Meyer test specificity on jeans. Sci Justice. 2019;59(3):359-361. 